\section{Determination of visual prognosis in children with open globe injuries}

\begin{abstract}
Aims To determine the predictive factors of visual outcomes in children with open globe injury and to give guidance to reduce the incidence of open globe injury.

Methods One hundred and forty eyes of 137 consecutive open globe injury patients, who were treated at the Eye Center of Second Bethune Hospital affiliated with Jilin University between August 2005 and August 2012, were retrospectively analyzed. Data recorded included demographic characteristics, causes of injury, location and extent of injury, presenting visual acuity, detailed ocular anterior and posterior segment evaluations, details of primary and subsequent surgeries, and postoperative complications and outcomes. The follow-up data included the most recent best-corrected visual acuity, complications, and the duration of follow-up.

Results Of the 137 patients, there were 116 (84.7\%) boys and 21 (15.3\%) girls. Their ages ranged between 3 and 17 years old (mean $=11.57 \pm 4.19$ years old). Sixty $(43.8 \%)$

children had a right eye injury, whereas 74 $(54.0 \%)$ had a left eye injury. Only three (2.2\%) children suffered bilateral eye injury. Living utensils, industrial tools, and fireworks contributed to the most common causes of open globe injury. Eighty-one $\mathbf{5 9 . 1 \%}$ ) had sharp force injuries, $23(\mathbf{1 6 . 8 \%})$ had blunt injuries, and $33(24.1 \%)$ had missile injuries. Conclusions Unfavorable visual outcomes were related to a younger age at presentation, poor presenting visual acuity, injuries caused by blunt or missile objects, posterior wound location, hyphema, vitreous hemorrhage, and surgical intervention of pars plana vitrectomy. Eye (2014) 28, 852-856; doi:10.1038/eye.2014.108; published online 16 May 2014
\end{abstract}

Department of Ophthalmology, The Second Hospital of Jilin University, Changchun, China

Correspondence:

G Su or J Zhao,

Department of

Ophthalmology, The

Second Hospital of Jilin

University, \#218 Ziqiang

Street, Changchun, Jilin

130021, China

Tel: +86431 89575898 or +8643189575899;

Fax: +86 43189575899 .

E-mail: sugf2012@

163.com or

jinsongzhao2003@

163.com

Received: 5 December 2013 Accepted in revised form: 25 March 2014

Published online: 16 May 2014

\section{Introduction}

Open globe injury (OGI) may lead to irreversible damage to the intraocular tissues and is a major cause of noncongenital monocular blindness in children. ${ }^{1-3}$ It has been reported that approximately $43 \%$ cases occur in people younger than 18 years of age. ${ }^{4}$ Incomplete or even inaccurate injury descriptions, as well as poor cooperation during the ocular examination and postoperative follow-up make it difficult to evaluate the prognosis of pediatric OGI. ${ }^{3,4}$ The influencing factors that make the management and prognosis determination more complex also include an incompletely developed visual system, active inflammatory response, and exuberant wound healing together with amblyopia. ${ }^{5}$

It has been shown that visual prognostic factors of OGI in adults include presenting visual acuity (VA), types of trauma, location and length of wounds, lens damage, vitreous hemorrhage $(\mathrm{VH})$, and types of intraocular foreign bodies, and so on.6,7 Relatively little is known regarding the visual prognostic factors in children. ${ }^{8,9}$ Despite the high incidence and probability of causing blindness, it is a preventable and controllable disease.

\section{Materials and methods}

This retrospective case series enrolled OGI patients of age 17 years or younger who were treated at the Eye Center of Second Bethune Hospital affiliated with Jilin University between August 2005 and August 2012. OGI was defined as any traumatic and full-thickness wound of the globe (injury of the sclera, cornea, or both). ${ }^{10}$ Mechanisms were classified as blunt, sharp, or missile. ${ }^{7}$ The location and extent of the injury were defined according to the Ocular Trauma Classification Group: zone I, isolated to the cornea or corneoscleral limbus; zone II, limited to the anterior $5 \mathrm{~mm}$ of sclera; zone III, fullthickness injury $>5 \mathrm{~mm}$ beyond the limbus. ${ }^{10}$ Patients who were followed up for at least 3 months were included. Poor visual outcome 
was defined as a final best corrected visual acuity (BCVA) $<20 / 200$.

Data recorded included demographic characteristics, cause of injury, mechanism of injury, location and extent of injury, presenting VA, clinical signs (the presence of a relatively afferent pupillary defect, hyphema, uveal tissue or vitreous prolapse, traumatic cataract, $\mathrm{VH}$, endophthalmitis, retinal detachment (RD), or an intraocular foreign body), details of primary and subsequent surgeries, and postoperative complications and visual outcomes. Follow-up data, including most recent $\mathrm{BCVA}$, complications, and duration of follow-up, were collected.

All corneal wounds were sutured with 10/0 ethilon (Johnson \& Johnson Medical Ltd., St Stevens-Woluwe, Belgium), whereas scleral wounds were sutured with $6 / 0$ vicryl (Johnson \& Johnson Medical Ltd.) or 5/0 PremiCron (B Braun Surgical S.A, Barcelona, Spain). Necrotic or infected prolapsed uveal tissues were excised, and the prolapsed vitreous was cut by a vitrectomy. Lens aspiration was performed in eyes with lens capsule rupture. Intravitreal vancomycin was injected when an infection was suspected to exist. Intravenous antibiotics were given before and after the surgical intervention in some cases. Secondary operations required in some patients included anterior chamber washout, intraocular lens implantation, and pars plana vitrectomy (PPV), and so on.

Either the $\chi^{2}$-test or the Fisher's exact test was used to investigate the association between two categorical variables. For multivariate analysis, stepwise logistic regression was conducted to determine the predictive variables for the final visual outcome. A $P$-value less than 0.05 indicated statistical significance. SPSS version 17.0 (SPSS, Inc., Chicago, IL, USA) was used for the statistical analyses.

\section{Results}

One hundred and thirty-seven OGI patients (140 eyes) younger than 17 years of age, including 116 (84.7\%) boys and $21(15.3 \%)$ girls were recruited in this study. Their mean age was $11.57 \pm 4.19$ years (range: $3-17$ years; Figure 1). Sixty (43.8\%) patients had a right eye injury and $74(54.0 \%)$ had a left eye injury. Bilateral ocular injuries occurred in three $(2.2 \%)$ children. Injuries occur most frequently in February (16.8\%) when spring festival is celebrated and August (12.4\%) which is during the summer vacation (Figure 2 ). The causes of injuries were summarized in Tables $1 \mathrm{a}$ and $1 \mathrm{~b}$. One hundred and twenty $(87.6 \%)$ children went to the hospital within $24 \mathrm{~h}$ of injury and $17(12.4 \%)$ waited for 1 to 11 days before going to the hospital. Table 2 showed the distribution of the presenting VA and final BCVA.
Among the eyes with presenting VA better than HM, there was no RD found in the presenting or follow-up period. The mean follow-up period was 9.6 months.

The predictive factors of the final BCVA was shown in Table 3. Higher rate of preschool-aged children (70.0\%) and injuries caused by blunt subjects $(73.9 \%)$ had a final BCVA worse than 20/200. Poor visual outcome was significantly associated with younger age and injuries due to blunt objects $(P=0.024, P=0.027)$.

Eyes with a poor preoperative VA tended to have a worse visual outcome $(P<0.001)$. Sixty-nine $(65.1 \%)$ eyes that had a presenting VA worse than $20 / 200$ had a poor visual outcome, whereas only $2(5.9 \%)$ of the 34 eyes with a better preoperative vision had the same consequence. By multivariate analysis, a presenting VA of $<20 / 200$ was determined to be an independent predictive factor for a final BCVA of $<20 / 200(P<0.001, \mathrm{OR}=31.905,95 \%$ $\mathrm{CI}=6.942-146.643)$.

A much lower rate of unsatisfactory final BCVA was achieved in cornea wound $(39.4 \%)$ compared with the zone $2(61.9 \%)$ and zone $3(63.0 \%)$. Posterior wound correlated significantly with a poor final VA $(P=0.007)$. By multivariate analysis, a wound located in zone 2 or 3 was determined to be an independent predictive factor for a final VA $<20 / 200(P=0.026, \mathrm{OR}=0.398,95 \%$ $\mathrm{CI}=0.177-0.897)$

Nineteen $(70.4 \%)$ eyes with hyphema at the time of the initial examination did not achieve a satisfactory VA. In contrast, only $52(46.0 \%)$ eyes had a poor visual outcome in eyes without hyphema. This result was statistically significant between the two groups $(P=0.023)$.

Eleven eyes with $\mathrm{VH}$ and seven eyes with RD were noted. Only two $(28.2 \%)$ eyes with VH were found to have a VA of 20/200 or better. All RD eyes had a poor visual outcome. Eyes with $\mathrm{VH}$ tended to have a worse visual outcome $(P=0.032)$. Although statistical significance was achieved between a poor visual outcome and the presence of RD, more cases are needed to obtain a more accurate conclusion.

All 140 injured eyes received primary repair on the day of presentation. PPV was performed on 17 eyes, including primary and subsequent operations, in which 14 eyes had a presenting VA of HM to NLP. Fifteen eyes had a final VA better than CF and three eyes even achieved VA better than 20/50. Forty-six (32.9\%) eyes had secondary surgeries, including procedures treating hyphema, traumatic cataracts, aphakic conditions, endophthalmitis, $\mathrm{VH}$, and RD. A poor visual outcome might be related to the need for a vitrectomy $(P=0.005)$, but not to the numbers of operations performed $(P=0.809)$.

Delay of presentation $(n=17)$, iris prolapse $(n=13)$, lens injury $(n=61)$, a foreign body in the posterior segment of 




Figure 1 The graph shows the age and sex distribution of the children with open globe injury at presentation. One hundred and thirty-seven pediatric open globe injury patients were recruited, including 116 boys and 21 girls, ranging from 3 to 17 years of age.

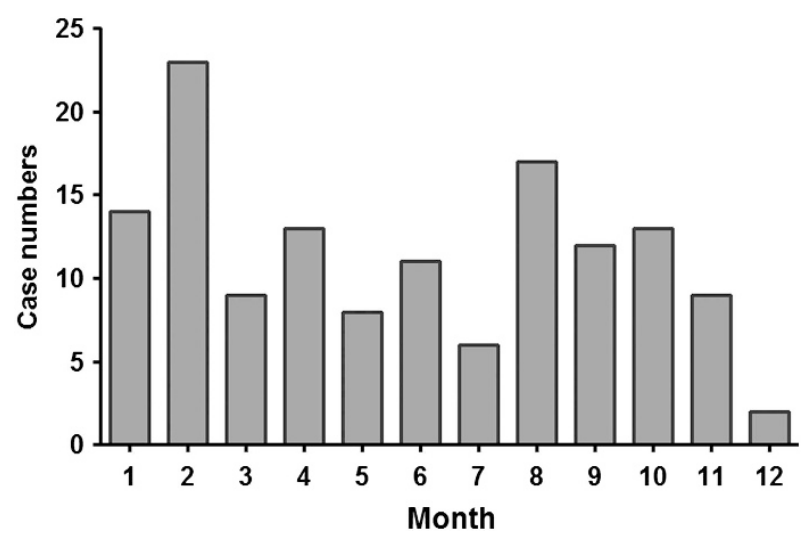

Figure 2 The graph shows the distribution of injuries by calendar month. Injuries occurred most frequently in February and August.

Table 1a Summary of the mechanisms of injury sorted by the type

\begin{tabular}{lc}
\hline Mechanisms & Number and proportion \\
\hline Household supplies & $46(33.6 \%)$ \\
Tools & $25(18.2 \%)$ \\
Firecrackers & $25(18.2 \%)$ \\
Stationary and toy & $9(6.6 \%)$ \\
Detonators & $7(5.1 \%)$ \\
Glasses & $3(2.2 \%)$ \\
Others & $17(12.5 \%)$ \\
Unknown & $5(3.6 \%)$ \\
\hline
\end{tabular}

the eye $(n=18)$, and endophthalmitis $(n=18)$ were not found to be predictive factors of poor visual outcomes.

\section{Discussion}

OGI is usually difficult to assess and prone to induce severe visual impairment or even visual loss, especially in children. In the current study, we investigated the
Table $\mathbf{1 b}$ Summary of the mechanisms of injury sorted by the character

\begin{tabular}{lc}
\hline Mechanisms & Number and proportion \\
\hline Metallic & $40(29.2 \%)$ \\
Explosive & $32(23.4 \%)$ \\
Glass & $17(12.4 \%)$ \\
Plastic and resin & $13(9.5 \%)$ \\
Animal & $12(8.8 \%)$ \\
Wooden & $10(7.3 \%)$ \\
Others & $8(5.8 \%)$ \\
Unknown & $5(3.6 \%)$ \\
\hline
\end{tabular}

Table 2 Distribution of the PVA and final BCVA

\begin{tabular}{lrcccc}
\hline VA & NLP & LP/HM & $1 / 200-19 / 200$ & $20 / 200-20 / 50$ & $\geq 20 / 40$ \\
\hline Presenting VA & 13 & 59 & 29 & 31 & 8 \\
Final BCVA & 6 & 23 & 28 & 47 & 36
\end{tabular}

Abbreviations: BCVA, best-corrected visual acuity; HM, hand move; LP, light perception; NLP, no light perception; VA, visual acuity.

predictive factors of visual outcomes in children, hoping to give guidelines in treatment planning and visual expectation counseling.

It has been reported that boys often have a greater incidence than girls, ranging from $2: 1$ to $6: 1.3,7,11$ In this case series, the boy-girl ratio was $5.5: 1$. Our average age of onset was 11.57 years. Although some studies have shown an even distribution between the age groups $s^{3,9}$ or a younger age of onset, ${ }^{1,6}$ teenagers in the present study involved more in the trauma, probably due to their increased participation in social activities and labor work, with less surveillance and protective measures.

The preschool-aged group had the greatest percentage of children with a poor final BCVA in this study, which is similar to previous studies. ${ }^{1,12,13}$ In Hill's study, however, children in the age group of 9-17 years tended to have more serious injuries and worse visual outcomes. ${ }^{6}$ Of the different age groups, the causes and locations of trauma together with other conditions (such as an intraocular foreign body, $\mathrm{VH}$, and so on) are different, but all these factors are reported to be predictive factors of pediatric OGI. 3,6,9 Amblyopia is also a main reason of the poor visual outcome in young children with OGI. ${ }^{7}$ The visual prognosis should not depend merely on the age of onset, but it should be combined with a comprehensive analysis. For young children, attention should be paid to their follow-up to prevent or correct the factors that may lead to amblyopia or strabismus.

Sharp injuries were the major cause in this case series, causing less visual impairment than the other causes, which is similar to the series of Gupta et al and other studies. $1,7,8,11,13$ Wounds due to sharp injuries are more often located in the cornea, whereas those due to blunt 
Table 3 Predictive factors of the final BCVA

\begin{tabular}{|c|c|c|c|c|}
\hline Variable & Teams & $\begin{array}{c}\text { Final BCVA } \\
\geq 20 / 200\end{array}$ & $\begin{array}{c}\text { Final BCVA } \\
<20 / 200\end{array}$ & P-value \\
\hline \multirow[t]{3}{*}{ Age (years) } & $<6$ & $6(30.0 \%)$ & $14(70.0 \%)$ & $0.024^{*}$ \\
\hline & $6-11$ & $30(63.8 \%)$ & $17(36.2 \%)$ & \\
\hline & $12-17$ & $33(45.2 \%)$ & $40(54.8 \%)$ & \\
\hline \multirow[t]{2}{*}{ Presenting time $(\mathrm{h})$} & $<24$ & $58(45.7 \%)$ & $65(52.8 \%)$ & 0.175 \\
\hline & $\geq 24$ & $11(64.7 \%)$ & $6(35.3 \%)$ & \\
\hline \multirow[t]{3}{*}{ Mechanism of injury } & Sharp & $47(57.3 \%)$ & $35(42.7 \%)$ & \\
\hline & Blunt & $6(26.1 \%)$ & $17(73.9 \%)$ & $0.027^{*}$ \\
\hline & Missile & $16(45.7 \%)$ & $19(54.3 \%)$ & \\
\hline \multirow[t]{2}{*}{ Presenting VA } & $\geq 20 / 200$ & $32(94.1 \%)$ & $2(5.9 \%)$ & $<0.001^{*}$ \\
\hline & $<20 / 200$ & $37(34.9 \%)$ & $69(65.1 \%)$ & \\
\hline \multirow[t]{2}{*}{ Wound location } & Zone 1 & $43(60.6 \%)$ & $28(39.4 \%)$ & $0.007^{*}$ \\
\hline & Zone 2 and 3 & $26(37.7 \%)$ & $43(62.3 \%)$ & \\
\hline \multirow[t]{2}{*}{ Iris prolapse } & Yes & $6(46.2 \%)$ & $7(53.8 \%)$ & 0.813 \\
\hline & No & $63(49.6 \%)$ & $64(50.4 \%)$ & \\
\hline \multirow[t]{2}{*}{ Hyphema } & Yes & $8(29.6 \%)$ & $19(70.4 \%)$ & $0.023^{*}$ \\
\hline & No & $61(54.0 \%)$ & $52(46.0 \%)$ & \\
\hline \multirow[t]{2}{*}{ Lens injury } & Yes & $32(52.5 \%)$ & $29(47.5 \%)$ & 0.509 \\
\hline & No & $37(46.8 \%)$ & $42(53.2 \%)$ & \\
\hline \multirow[t]{2}{*}{ Posterior foreign body } & Yes & $6(33.3 \%)$ & $12(66.7 \%)$ & 0.147 \\
\hline & No & $63(51.6 \%)$ & $59(48.4 \%)$ & \\
\hline \multirow[t]{2}{*}{ Vitreous hemorrhage } & Yes & $2(28.2 \%)$ & $9(81.8 \%)$ & $0.032^{*}$ \\
\hline & No & $67(51.9 \%)$ & $62(48.1 \%)$ & \\
\hline \multirow[t]{2}{*}{ Retinal detachment } & Yes & $0(0.0 \%)$ & $7(100.0 \%)$ & $0.013^{\mathrm{a}}$ \\
\hline & No & $69(51.9 \%)$ & $64(48.1 \%)$ & \\
\hline \multirow{2}{*}{ Endophthalmitis } & Yes & $8(44.4 \%)$ & $10(55.6 \%)$ & 0.660 \\
\hline & No & $61(50.0 \%)$ & $61(50.0 \%)$ & \\
\hline \multirow[t]{2}{*}{ Multiple surgeries } & Yes & $22(47.8 \%)$ & $24(52.2 \%)$ & 0.809 \\
\hline & No & $47(50.0 \%)$ & $47(50.0 \%)$ & \\
\hline \multirow[t]{2}{*}{ Vitrectomy performed } & Yes & $3(17.6 \%)$ & $14(82.4 \%)$ & $0.005^{*}$ \\
\hline & No & $66(53.7 \%)$ & $57(46.3 \%)$ & \\
\hline
\end{tabular}

Abbreviations: BCVA, best-corrected visual acuity; VA, visual acuity. $* P<0.05$.

although $P<0.05$, more cases are needed to reach a more accurate conclusion.

and projectile injuries are usually long or irregular and with a greater incidence of intraocular foreign bodies, which would affect the visual prognosis.

The presenting VA is a strong prognostic indicator of visual outcome not only in adults but also in children with OGI. ${ }^{7,11,14-16}$ Our study showed similar results to those found in most studies, which indicate that a presenting $\mathrm{VA}<20 / 200$ is an independent risk factor of a final $B C V A<20 / 200$. Although some studies have found that a primary BCVA of $20 / 400$ or worse could not be a good predictor of a poor final visual outcome considering the probability of incompliance during examination or improvements in concept and technology of surgical intervention, 6,13 the presenting VA of HM or worse has been reported to be a risk factor of RD and hypotony at follow-up exams, ${ }^{17,18}$ which could be also found in our study.

The cornea is the most frequently involved structure in OGI because of its anterior location. Wounds limited to the cornea have been reported to be associated with a good visual outcome, unless the injury involved the visual axis or greater than $25 \%$ of the corneal diameter. ${ }^{15}$ Posterior wound location may cause severe damage to the photoreceptors such as the retina and optic nerve, thus indicating a poor prognosis. ${ }^{19}$ Children would develop serious inflammation, proliferation, and traction retinal detachment. ${ }^{20}$ In this case series, all of the seven eyes developing RD were injured on zone 2 or 3 , and the final BCVA values were 20/200 or worse.

Hyphema is another sign of an unfavorable visual prognosis. ${ }^{1,9}$ Gupta et al mentioned in a child-based study that the absence of the red reflex could reflect the severity of the injury, and hyphema was the main reason of the absence of a red reflex. ${ }^{7}$ We found that eyes with hyphema at the time of the initial examination were not prone to achieve a satisfactory VA. Meanwhile, presence of $\mathrm{VH}$ had some prognostic value. ${ }^{1,9} \mathrm{VH}$ might be caused by rupture of the blood vessels in the ciliary body, retina, urea, or sclera, and it could be related to more serious damage of the eye tissues and unfavorable visual outcome.

RD in OGI which could be induced by direct trauma or traction of proliferative vitreous, often needs multiple surgeries and is associated with a poor prognosis, or even pthisis. Many studies have confirmed the association between RD and poor prognostic features. ${ }^{1,9,11,12}$ Photoreceptor cells are seriously injured when RD occurs, and proliferative vitreoretinopathy after RD is another factor that may cause limited postoperative vision.

PPV was found to be related to a poor visual outcome in this study, but some improvements of visual outcome were finally obtained. PPV can help a lot in treating the posterior segment injury and lens trauma, including removing blood or foreign body in vitreous, reducing tissue damage due to inflammation and toxins, releasing the vitreous retraction, reducing proliferation by removing the support for cell migration, and promoting anatomical and functional recovery of injured eyes, especially for severe ocular trauma. 3,11 With the advances of the technology, PPV has an important role in not only management of injured eyes but also improving the visual outcomes.

\section{Conclusion}

Our findings showed that the prognostic factors for a VA worse than 20/200 included an age less than 6 years at presentation, poor presenting VA, injuries caused by blunt or missile objects, posterior wound location, hyphema, VH, and surgical intervention of PPV. A poor presenting VA together with a posterior wound location were independent risk factors in the multivariate analysis. As a result, the prognostic factors for poor visual outcomes might be used to evaluate injuries and determine the appropriate therapy. 


\section{Summary}

\section{What was known before}

- The usual causes of open globe injuries in children are knives, scissors, and so on.

- More boys are involved.

- It is difficult to evaluate the prognosis of pediatric OGI cases.

- Satisfied visual outcome in children: sharp injuries, eyes without hyphema.

- Other factors are still in debate.

What this study adds

- Situations of pediatric open globe injuries in China, especially the northeast part of China.

- Conditions of surgical intervention including the pars plana vitrectomy in treating open globe injuries.

- The most common causes of injuries: utensils of daily life, industrial tools, fireworks.

- Prognostic factors for a VA worse than 20/200: age of 0-6 years old, Poor PVA, injuries caused by blunt or missile objects, posterior wound location, hyphema, vitreous hemorrhage, multiple surgical interventions.

- Independent risk factors in the multivariate analysis: poor presenting visual acuity, posterior wound location.

\section{Conflict of interest}

The authors declare no conflict of interest.

\section{Acknowledgements}

This work was supported by the National Natural Science Foundation of China [31071222] and the Frontier Interdiscipline Program of Norman Bethune Health Center of Jilin University [2013106023].

\section{References}

1 Rostomian K, Thach AB, Isfahani A, Pakkar A, Pakkar R, Borchert M. Open globe injuries in children. J AAPOS 1998; 2: 234-238.

2 Serrano JC, Chalela P, Arias JD. Epidemiology of childhood ocular trauma in a northeastern Colombian region. Arch Ophthalmol 2003; 121: 1439-1445.

3 Tok O, Tok L, Ozkaya D, Eraslan E, Ornek F, Bardak Y. Epidemiological characteristics and visual outcome after open globe injuries in children. J AAPOS 2011; 15: 556-561.
4 Alfaro DV, Chaudhry NA, Walonker AF, Runyan T, Saito Y, Liggett PE. Penetrating eye injuries in young children. Retina 1994; 14: 201-205.

5 Ference Kuhn DJP. Ocular trauma: principles and practice. Thieme New York: New York, 2010.

6 Hill JR, Crawford BD, Lee H, Tawansy KA. Evaluation of open globe injuries of children in the last 12 years. Retina 2006; 26: S65-S68.

7 Gupta A, Rahman I, Leatherbarrow B. Open globe injuries in children: factors predictive of a poor final visual acuity. Eye (Lond) 2009; 23: 621-625.

8 Pieramici DJ, MacCumber MW, Humayun MU, Marsh MJ, de Juan Jr E. Open-globe injury. Update on types of injuries and visual results. Ophthalmology 1996; 103(11): 1798-1803.

9 Lee CH, Lee L, Kao LY, Lin KK, Yang ML. Prognostic indicators of open globe injuries in children. Am J Emerg Med 2009; 27: 530-535.

10 Pieramici DJ, Sternberg Jr P, Aaberg Sr TM, Bridges Jr WZ, Capone Jr A, Cardillo JA et al. A system for classifying mechanical injuries of the eye (globe). The Ocular Trauma Classification Group. Am J Ophthalmol 1997; 123: 820-831.

11 Farr AK, Hairston RJ, Humayun MU, Marsh MJ, Pieramici DJ, MacCumber MW et al. Open globe injuries in children: a retrospective analysis. J Pediatr Ophthalmol Strabismus 2001; 38: 72-77.

12 Sheard RM, Mireskandari K, Ezra E, Sullivan PM. Vitreoretinal surgery after childhood ocular trauma. Eye (Lond) 2007; 21: 793-798.

13 Bunting H, Stephens D, Mireskandari K. Prediction of visual outcomes after open globe injury in children: a 17-year Canadian experience. J AAPOS 2013; 17(1): 43-48.

14 Kuhn F, Morris R, Witherspoon CD, Heimann K, Jeffers JB, Treister G. A standardized classification of ocular trauma. Ophthalmology 1996; 103: 240-243.

15 Baxter RJ, Hodgkins PR, Calder I, Morrell AJ, Vardy S, Elkington AR. Visual outcome of childhood anterior perforating eye injuries: prognostic indicators. Eye (Lond) 1994; 8: 349-352.

16 Sternberg Jr P, de Juan Jr E, Michels RG, Auer C. Multivariate analysis of prognostic factors in penetrating ocular injuries. Am J Ophthalmol 1984; 98: 467-472.

17 Al-Mezaine HS, Osman EA, Kangave D, Abu El-Asrar AM. Prognostic factors after repair of open globe injuries. J Trauma 2010; 69: 943-947.

18 Entezari M, Rabei HM, Badalabadi MM, Mohebbi M. Visual outcome and ocular survival in open-globe injuries. Injury 2006; 37: 633-637.

19 Man CY, Steel D. Visual outcome after open globe injury: a comparison of two prognostic models-the Ocular Trauma Score and the Classification and Regression Tree. Eye (Lond) 2010; 24: 84-89.

20 Scott IU, Flynn Jr HW, Azen SP, Lai MY, Schwartz S, Trese MT. Silicone oil in the repair of pediatric complex retinal detachments: a prospective, observational, multicenter study. Ophthalmology 1999; 106: 1399-1407; discussion 407-408. 